\title{
MINI-REVIEW
}

\section{Pathomechanism of malignant melanoma}

\section{Nakamura Motonobu}

Department of Dermatology, School of Medicine, University of Occupational and Environmental Health, Kitakyushu 807-8555, Japan. E-mail: motonaka@med.uoeh-u.ac.jp

\begin{abstract}
Treatment of malignant melanoma has made great strides in the last decade. On the one hand, immune checkpoint inhibitors and molecular targeted drugs improved the prognosis of patients. On the other hand, it is very important to be aware of the causes of malignant melanoma based on the latest knowledge. In this review, I will briefly review the carcinogenic mechanism of malignant melanoma from the aspects of ultraviolet rays, mechanical stress, trauma and so on. Keywords: Malignant Melanoma; Mutation; Ultraviolet Ray; Pathomechanism
\end{abstract}

\section{ARTICLE INFO}

Article history:

Received 25 July 2021

Received in revised form 5 August 2021

Accepted 22 August 2021

Available online 26 August 2021

\section{COPYRIGHT}

Copyright (C) 2021 Nakamura Motonobu. doi: 10.24294/ti.v5.i1.1280

EnPress Publisher LLC. This work is licensed under the Creative Commons Attribution-NonCommercial 4.0 International License (CC BY-NC 4.0).

https://creativecommons.org/licenses/by-nc/ $4.0 /$

\section{Introduction}

The treatment of malignant melanoma has changed significantly with the launch of immune checkpoint inhibitors ${ }^{[1,2]}$. There are also reports on appropriate drug therapy ${ }^{[3]}$ and measures against side effects, and safer and more effective treatment methods are being pursued ${ }^{[4-6]}$. For that purpose, it is necessary to update the cause of malignant melanoma.

Ultraviolet light (UVL) is the oldest and most well-known cause of malignant melanoma. It is widely known that the incidence of malignant melanoma is significantly higher in Caucasians than in colored races including Asians, and awareness-raising activities for UV protection are currently being actively carried out, especially in Europe and the United States. Recently, it has been pointed out that external force, trauma, radiation, etc. may also trigger malignant melanoma.

In this paper, I would like to outline the causes or triggers of these malignant melanomas, including some basic data.

\section{UV rays and malignant melanoma}

In the living body, when exposed to UV rays, pyrimidine dimers (Figure 1) and 6-4 photoproducts (Figure 2) are produced ${ }^{[7]}$, and the mutation of cytosine to thymine (CT mutation) occurs, if repair systems do not work (Figure 3).

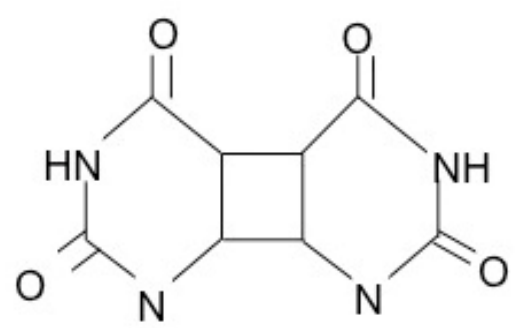

Figure 1. Pyrimidine dimer produced by ultraviolet rays. 


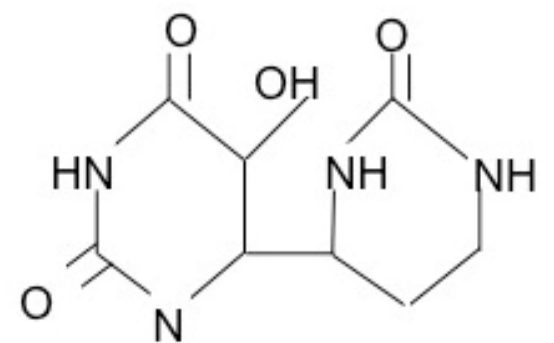

Figure 2. 6-4 photoproducts produced by ultraviolet rays.

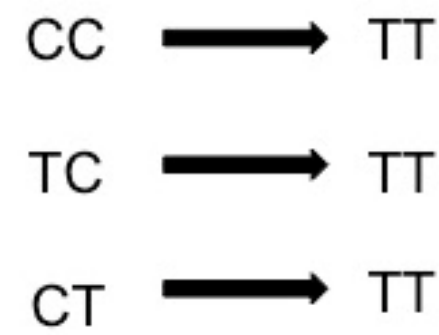

Figure 3. Cytosine to thymine mutation caused by ultraviolet rays (CT mutation).

Normally, when pyrimidine dimer or 6-4 photoproduct is produced, UV-specific endonuclease recognizes and incises DNA, DNA polymerase replicates DNA from the normal side, DNA ligase implants, and repair is completed.

A disease in which UV repair function is congenitally impaired is xeroderma pigmentosum (XP), which is an autosomal recessive inheritance disease. Patients with XP develop erythema when exposed to solar UV rays from an early age, and often develop malignant melanoma, squamous cell carcinoma (SCC), basal cell carcinoma (BCC), etc. at an early age.

Among UV rays, medium-wave UVB (ultraviolet B) has been considered to have a strong carcinogenic effect, but recently, it has become clear that long-wave UVA (ultraviolet A) is also involved in carcinogenesis. Skin cancer is more likely to occur in exposed areas than in non-exposed areas, and within the same ethnic group, the incidence is higher in the equatorial region where the sun's rays are stronger. In addition, skin cancer occurs more frequently in people working outdoors than indoors, and the risk of skin cancer is inversely correlated with the degree of melanin pigmentation. From these results, it is considered that solar UV rays are deeply involved in skin malignant tumors such as SCC, BCC, and malignant melanoma, and the number of patients with any skin malignant tumor increased due to ozone layer depletion.

Malignant melanoma often occurs in exposed areas in Europe and the United States, and it is thought that UV rays are involved. In Japan, the number of cases is high in the southern regions in terms of latitude, in which the primary site is $14 \%$ on the face and head, and $27 \%$ on the sole, which does not necessarily occur on the exposed area. It is speculated that the number of acral types will increase because the amount of melanin in the skin of the yellow race is larger than Caucasian and its protective ability is associated with relative low number of UV-induced malignant even if the number of cases is the same.

\section{Model animal for malignant me- la-noma}

In 1989, Setlow et al. ${ }^{[8]}$ exposed zebrafish to visible light to make the first model animal for malignant melanoma. In 2002, Noonan et al. ${ }^{[9]}$ succeeded to develop malignant melanoma in the epidermis of irradiate hepatocyte growth factor (HGF) transgenic mice with UVB + UVA at 3, 5, and 6 weeks after birth.

In 1998, Kunisada et al. ${ }^{[10]}$ linked c-kit under the keratin 14 promoter to overexpress stem cell factor (SCF) in the epidermis, and created transgenic mice with mast cell proliferation and melanocytes present in the epidermis. By crossing this mouse with an XPA knockout mouse, a mouse having high UV sensitivity and having melanocytes in the epidermis was created ${ }^{[11]}$. In this mouse, when a large amount of UVB was irradiated, malignant melanoma could be generated in the epidermis even when it was not a child, and it became a mouse model closer to human malignant melanoma.

Thus, these results suggest that UV rays are essentially involved in the development of malignant melanoma.

\section{Foot sole melanoma and mecha- nical stress}

As mentioned above, it has become clear that UV rays are closely related to the development of malignant melanoma, but malignant melanoma does not always occur in exposed areas, and factors other 
than UV rays are also involved. The sole of the foot is a common site for malignant melanoma in Japanese as well as Asian people, in which the amount of UV exposure is low. The effects of mechanical stress on sole malignant melanoma were investigated at Shinshu University, Japan ${ }^{[12]}$.

Of the malignant melanomas treated at Shinshu University, Japan from 1990 to 2014, 123 cases with lesions on the sole of the foot were included, and cases originating from the nails were excluded. Detailed lesion locations were compared using clinical photographs, and resected specimens measured tumor thickness on a Breslow scale. Of the 123 cases, 54 were male and 69 were female, with an average age of 73.5 years. It occurred on the left sole in 61 cases and on the right sole in 62 cases. The number of lesions by tumor thickness on the Breslow scale was 28 for in situ, 12 for $1 \mathrm{~mm}$ or less, 20 for $1.01 \mathrm{~mm}$ to $2 \mathrm{~mm}, 28$ for $2.01 \mathrm{~mm}$ to 4 $\mathrm{mm}, 33$ for $4.01 \mathrm{~mm}$ or more, and 2 for unmeasurable. Analysis of mapping the center of the lesions revealed that the most lesions were distributed in the heel, followed by the $1^{\text {st }}$ and $5^{\text {th }}$ toes. In addition, there were almost no lesions on the arch. There was no bias in the distribution of lesions depending on the tumor thickness. Looking at the number of lesions per $1 \mathrm{~cm}^{2}$, many lesions were accumulated in the heel and toe, but there were almost no lesions in the arch. From these facts, it was speculated that mechanical stress due to load-bearing was involved in the pathophysiology of sole malignant melanoma.

\section{Trauma and subungual malig- nant melanoma}

There is a history of trauma in subungual malignant melanoma, and there are many cases in which trauma may be the trigger. According to the report by Takai et al. ${ }^{[13]}, 62 \%$ of all subungual malignant melanomas were accompanied by obvious trauma. Omoto et al. ${ }^{[14]}$ collected the literature on subungal malignant melanoma with a history of trauma, and the average time from trauma to diagnosis was 99 months for adults and children. For those who develop pigmented streaks as a result of trauma and subsequently develop malignant mela- noma, the average time to diagnosis is about 130 months for adults and 72 months for children. Besides, it has been reported that the time to onset of pigmented streaks with trauma is as short as 15 months in adults, and that it progresses rapidly after trauma in children.

In other words, subungual malignant melanoma appears earlier when trauma is applied to the part where the pigmented streak was originally present than where there is no pigmented streak.

\section{Radiation and malignant mela- noma}

Otsu et al. ${ }^{[15]}$ reported the proportion of cutaneous malignancies that developed after irradiation for 1923-1980 and 1981-2014, which were collected from Japanese literatures mainly. In 1923-1980, $62 \%$ had SCC, $12.6 \%$ had BCC, 3.7\% had intraepidermal cancer including Bowen's disease, and $0 \%$ had malignant melanoma. As well, in 1981-2014, $42 \%$ had SCC, $29.4 \%$ had BCC, $7.5 \%$ had intraepidermal cancer including Bowen's disease, and 2.5\% had malignant melanoma. That is, the proportion of BCC has markedly increased in recent years. The reason for this is that radiation was used for benign skin diseases including tumor diseases and chronic inflammatory diseases previously. The relative decrease in SCC was due to the improvement of site selection, irradiating dose reduction, and tangential irradiation application. Radiation-induced malignant melanoma is extremely rare compared to SCC, $\mathrm{BCC}$, and Bowen's disease, whether or not the slight increase of $2.5 \%$ makes sense.

\section{AID and malignant melanoma}

AID is an activation-induced cytidine deaminase (activation induced deaminase), a 24- kDa enzyme that removes an amino group from a cytidine group in DNA. In 2000, it was shown that this AID is essential for immunoglobulin class switching and point mutations in the variable region of immunoglobulin $^{[16]}$.

Moreover, it was hypothesized that AID is highly expressed in various malignant tumors such as liver cancer and gastric cancer, and that AID is induced by various inflammations and infections. 
Furthermore, the induction of a point mutation is speculated to be closely associated with carcinogenesis. When the expression of AID was examined in malignant melanoma, it was revealed that malignant melanoma with high expression of AID frequently causes $\mathrm{BRAF}^{\mathrm{V} 600 \mathrm{E}}$ mutation and has a poor $\operatorname{prognosis}^{[17]}$.

In addition, malignant melanoma spontaneously developed when AID was highly expressed in all organs under the promoter that expresses it in all organs ${ }^{[18]}$. From these reports, AID may be involved in the development of $\mathrm{BRAF}^{\mathrm{V} 600 \mathrm{E}}$ mutation and malignant melanoma.

\section{Conflict of interest disclosure}

None declared.

\section{References}

1. Furukawa F. The Nobel Prize in Physiology or Medicine 2018 was awarded to cancer therapy by inhibition of negative immune regulation. Trends in Immunotherapy 2018; 2. doi: 10.24294/ti.v2.i3.10 65

2. Seidel JA, Otsuka A, Kabashima K. Treating tumors with immune checkpoint inhibitors: Rationale and limitations. Trends in Immunotherapy 2017; 1(1): 2-9. doi: 10.24294/ti.v1.i1.20

3. Furukawa F. Effects of immune checkpoint inhibitors on cancer patients with preexisting autoimmune disease. Trends in Immunotherapy 2021; 5(1): 5-6. doi: 10.24294/ti.v5.i1.1250

4. Yano S, Okuno A, Furukawa F. Severe type of erythema multiforme (EM major) due to administration of Anti-PD-1 antibody drug. Trends in Immunotherapy 2021; 5(1): 1-4. doi: 10.24294/ti.v5.i1.12 47

5. Adachi E, Yokoyama E, Yamagami Y, et al. Bullous pemphigoid induced by nivolumab in a patient with malignant melanoma. Trends in Immunotherapy 2020; 4(1): 15-17. doi: 10.24294/ti.v4.i1.1210

6. Inaba $\mathrm{H}$, Ariyasu $\mathrm{H}$, Okuhira $\mathrm{H}$, et al. Endocrine dysfunctions during treatment of immune-checkpoint inhibitors. Trends in Immunotherapy 2020; 4(1): 18-26. doi: 10.24294/ti.v4.i1.606

7. Yamazaki F. Solar UV rays and skin cancer (in Japanese). The Japanese Journal of Dermatology 2014; 124(6): 1121-1126. doi: https://doi.org/10.14 924/dermatol.124.1121
8. Setlow RB, Woodhead AD, Grist E. Animal model for ultraviolet radiation-induced melanoma: Platyfish-swordtail hybrid. Proceedings of the National Academy of Sciences of the United States of America 1989; 86(22): 8922-8926. doi: 10.1073/pn as. 86.22.8922

9. Noonan FP, Recio JA, Takayama H, et al. Neonatal sunburn and melanoma in mice. Nature 2001; 413(6853): 271-272. doi: 10.1038/35095108

10. Kunisada T, Lu S, Yoshida H, et al. Murine cutaneous mastocytosis and epidermal melanocytosis induced by keratinocyte expression of transgenic stem cell factor. Journal of Experimental Medicine 1998; 187(10): 1565-1573. doi: 10.1084/jem.187.1 0.1565

11. Yamazaki F, Okamoto H, Matsumura Y, et al. Development of a new mouse model (xeroderma pigmentosum a-deficient, stem cell factor-transgenic) of ultraviolet B-induced melanoma. Journal of Investigative Dermatology 2005; 125(3): 521-525. doi: 10.1111/j.0022-202X.2005.23753.x

12. Minagawa A, Omodaka T, Okuyama R. Melanomas and mechanical stress points on the plantar surface of the foot. The New England Journal of Medicine 374: 2404-2406. doi: 10.1056/NEJMc1512354

13. Takai I, Katsuura J, Kubota Y. A case of malignant melanoma of the nail - Summary of reported cases caused by trauma (in Japanese). Japanese Journal of Clinical Dermatology 2006; 60(3): 282-284. doi: https://doi.org/10.11477/mf.1412100550

14. Omoto M, Hayama K, Kimura K, et al. Pediatric cases of acral lentiginous melanoma under the nail plate (in Japanese). Rinsho derma (Tokyo) 2019; 61(9): 1444-1448. doi: https://doi.org/10.18888/hi. 0000001555

15. Otsu M, Nagase $\mathrm{K}$, Inoue $\mathrm{T}$, et al. Multiple squamous cell cancers and intraepidermal malignancies caused by chronic radiation dermatitis (in Japanese). Practical Dermatology 2015; 37(10): 1001-1004. doi: https://doi.org/10.24733/J01268.2016019656

16. Muramatsu M, Kinoshita K, Fagarasan S, et al. Class switch recombination and hypermutation require activation-induced cytidine deaminase (AID), a potential RNA editing enzyme. Cell 2000; 102(5): 553-563. doi: 10.1016/s0092-8674(00)00078-7

17. Okura R, Yoshioka H, Yoshioka M, et al. Expression of AID in malignant melanoma with $\mathrm{BRAF}^{\mathrm{V} 600 \mathrm{E}}$ mutation. Experimental Dermatology 2014; 23(5): 347-348. doi: 10.1111/exd.12402

18. Nonaka $\mathrm{T}$, Toda $\mathrm{Y}$, Hiai $\mathrm{H}$, et al. Involvement of activation-induced cytidine deaminase in skin cancer development. The Journal of Clinical Investigation 2016; 126(4): 1367-1382. doi: 10.1172/JCI815 22 